Conclusions EUS with FNA is effective and safe for diagnosis of biopsy negative malignant esophageal strictures.

\section{IDDF2019-ABS-0115 ENDOSCOPIC ULTRASOUND GUIDED THROMBIN INJECTION- AN EMERGING THERAPY FOR VISCERAL ARTERY PSEUDOANEURYSM}

Ashok Jhajharia*, Rupesh Pokharna, Deepak Sharma, Mayank Ameta, Prachis Ashdhir. SMS Medical College and Hospital, Jaipur, India

\subsection{6/gutjnl-2019-IDDFabstracts. 154}

Background Visceral artery pseudoaneurysm is a rare and potentially life-threatening vascular entity with a high mortality rate, conventionally managed with digital subtraction angiography with coil embolization or surgery. However, in cases where angiographic coil embolization is not possible due to technical reasons, computerized tomography/ultrasonographyguided thrombin injection remains a viable option. In our study, we intend to highlight the role of endoscopic ultrasound (EUS)-guided thrombin injection in the management of abdominal visceral artery pseudoaneurysm, which is either inaccessible by endovascular route or has a high surgical risk of complication.

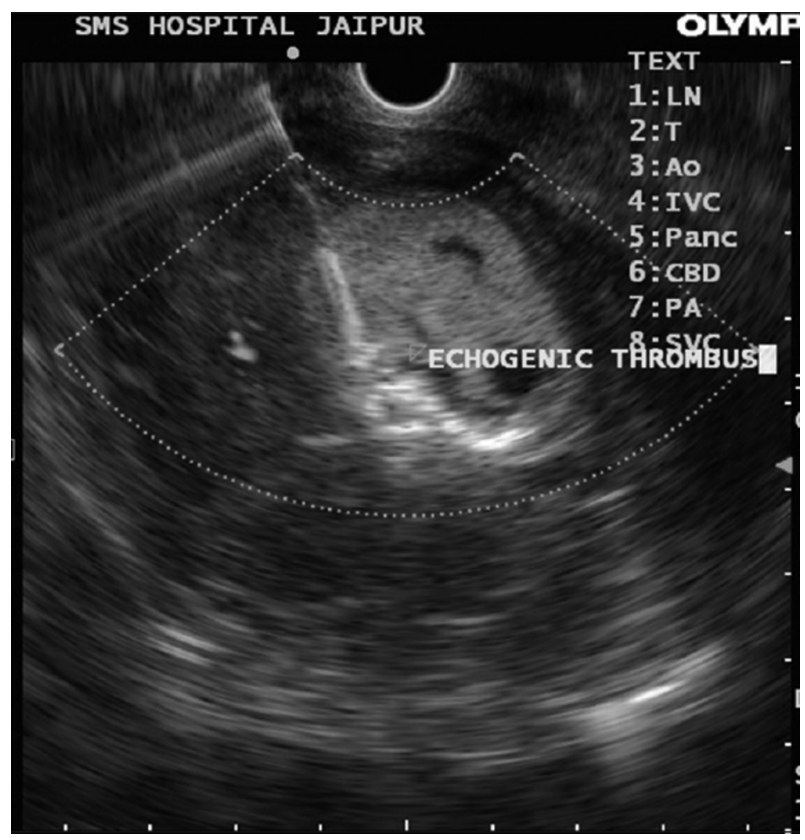

Abstract IDDF2019-ABS-0115 Figure 1

Aim To assess the efficacy of EUS guided thrombin injection in visceral artery pseudoaneurysm.

Methods A prospective study was conducted at SMS Hospital, Jaipur from June 2015 to January 2019. All patients with pseudoaneurysm with a history of gastrointestinal bleed were consecutively enrolled. Data relating to demography, laboratory parameters, radiological imaging, pseudo aneurysm and endotherapy were analyzed.

Results Sixteen patients with age 40 (25-58) years, 15 male and one female were studied. Etiology of pseudo aneurysm was chronic pancreatitis in $9(56 \%)$, acute pancreatitis in 2
(12.5\%), chronic liver disease in $2(12.5 \%)$, Blunt trauma abdomen in $2(12.5 \%)$ and idiopathic in $1(6 \%)$ patients. The site of pseudo aneurysm was splenic artery in $9(56 \%)$, hepatic artery in $4(25 \%)$ and gastroduodenal artery in 3 (19\%) patients. Median size of pseudo aneurysm was $2.2 \times 2.2$ $\mathrm{cm}(1.2 \times 1.8-3.5 \times 5.5 \mathrm{~cm})$ and neck size of artery was $2.6 \mathrm{~mm}(2.1-3.2 \mathrm{~mm})$. All patients presented with a history of hemetemesis and/or malena, two patients had hemobilia. Thrombin requirement was 400(300-1000) IU for loss of Doppler signals (figure 1). Computed tomography and EUS after 2 weeks, one month and 3 months showed an obliterated pseudo aneurysm. One patient required repeat thrombin injection after 2 weeks as there was an appearance of Doppler signal in pseudoaneurysm. One patient developed symptomatic splenic abscess after three weeks of procedure and required splenectomy. Technical success of procedure was $100 \%$.

Conclusions EUS-guided thrombin injection provides a new option for management of pseudoaneurysm especially in inaccessible by endovascular route and in patients having a high surgical risk.

\section{IDDF2019-ABS-0116 SELF-COMPLETION METHOD OF ENDOSCOPIC SUBMUCOSAL DISSECTION USING A NOVEL ENDO-KNIFE: AN EX VIVO PIG MODEL STUDY}

Mitsuru Esaki*, Toshiki Horii, Ryoji Ichijima, Sho Suzuki, Chika Kusano, Hisatomo Ikehara, Takuji Gotoda. Nihon University School of Medicine, Japan

\subsection{6/gutjnl-2019-IDDFabstracts. 155}

Background Endoscopic submucosal dissection (ESD) is a standard treatment for gastrointestinal tract tumors. Various endoscopic devices have been developed to improve the ESD procedure. We invented a self-completion ESD method using an Endosaber, which is a novel needle-type endo-knife for ESD (figure 1). Only one Endosaber was used without any additional device. In addition, ESD could be performed only by one operator without any assistance during the procedure. The aim of the study was to evaluate the technical outcomes of this method in an ex vivo pig training model.
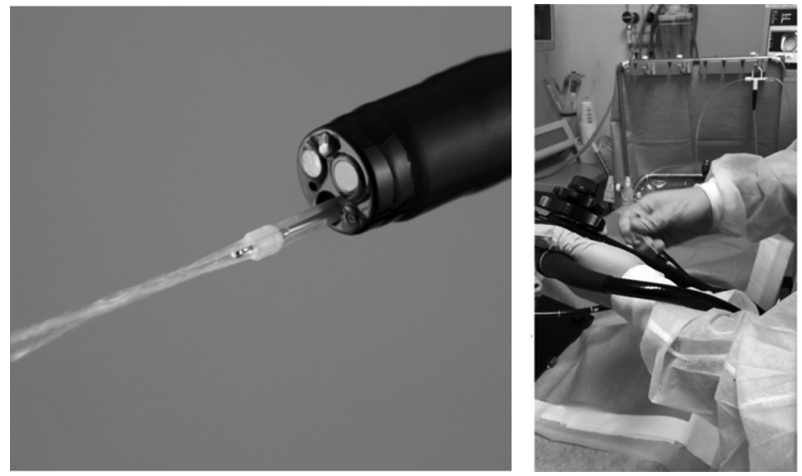

Abstract IDDF2019-ABS-0116 Figure 1

Methods This was a pilot study. ESD procedures were performed by four experts. Mock lesions of $15 \mathrm{~mm}$ diameter were made in the middle or lower esophagus of the porcine models. Each operator performed three ESDs on the mock 
lesions. The primary outcome was the completion rate of the self-completion ESD method. The secondary outcomes were the procedure time, en-bloc resection rate, perforation rate, and number of injections during the procedure.

Results In this study, all 12 cases of ESD were completed using the self-completion method (completion rate: 100\%). The median procedure time (range) was 483.5 (276-936) seconds [median incision time; 240.4 (98-605) seconds and median dissection time; 222 (137-617) seconds]. En-bloc and complete resection rates were $100 \%$ each. No perforation was noted during any of the procedures. The median number of injections was 10.5 (4-15). The procedure time significantly decreased with experience $(\mathrm{P}=0.0199)$.

Conclusions The self-completion ESD method using only one Endosaber without any assistance achieved a 100\% en-bloc resection rate without any perforation. Reduction in the need for an additional device or assistance was successful. This method may contribute to reducing the total cost of ESD procedure.

\section{IDDF2019-ABS-0118 ELECTROACUPUNCTURE PLUS ON-DEMAND GASTROCAINE FOR REFRACTORY FUNCTIONAL DYSPEPSIA: PRAGMATIC RANDOMIZED TRIAL}

${ }^{1}$ Vincent Chi Ho Chung ${ }^{*},{ }^{2}$ Charlene Hoi Lam Wong, ${ }^{3}$ Irene Xin Yin Wu, ${ }^{2}$ Jessica Yuet Ling Ching, 'William Kwok Wai Cheung, ${ }^{1}$ Benjamin Hon Kei Yip, ${ }^{4}$ Kam Leung Chan, ${ }^{2}$ Pui Kuan Cheong, ${ }^{2}$ Justin Che Yuen Wu. 'Jockey Club School of Public Health and Primary Care, The Chinese University of Hong Kong, Hong Kong; ${ }^{2}$ Department of Medicine and Therapeutics, The Chinese University of Hong Kong, Hong Kong; ${ }^{3}$ Xiang-Ya School of Public Health, Central South University, Chang-Sha, Hu-Nan, China; ${ }^{4}$ School of Chinese Medicine, The Chinese University of Hong Kong, Hong Kong

\subsection{6/gutjnl-2019-IDDFabstracts.156}

Background Treatment options for functional dyspepsia (FD) refractory to pharmacological treatments are limited, but the effectiveness of electroacupuncture (EA) is uncertain.

Our aim was to assess the effectiveness of EA combined with on-demand gastrocaine.

Methods We conducted a single-centre, assessor-blind, randomized parallel-group 2-arm trial on $H$. pylori negative FD patients of the postprandial distress syndrome (PDS) subtype refractory to proton pump inhibitor, prokinetics or $\mathrm{H}_{2}$ antagonists. Enrolled participants were block randomized in a 1:1 ratio, with concealed random sequence. The treatment and control groups both received on-demand gastrocaine for 12 weeks, but only those in treatment group were offered 20 sessions of EA over 10 weeks. The primary endpoint was the between-group difference in the proportion of patients achieving adequate relief of symptoms at week 12 .

Results Of 132 participants randomly assigned to EA plus ondemand gastrocaine $(n=66)$ or on-demand gastrocaine alone $(n=66), 125(94.7 \%)$ completed all follow-up at 12 weeks. The EA group had a compliance rate $97.7 \%$. They had a significantly higher likelihood in achieving adequate symptom relief at 12 weeks, with a clinically relevant number needed to treat (NNT) value of 2.36 (95\%CI: 1.74-3.64). Among secondary outcomes, statistically and clinically significant improvements were observed among global symptom $(\mathrm{NNT}=3.85(95 \% \mathrm{CI}: 2.63,7.69))$; postprandial fullness and early satiation ( $\mathrm{NNT}=5.00 \quad(95 \% \mathrm{CI}: 2.86,25.00))$; as well as epigastric pain, epigastric burning and postprandial nausea $(\mathrm{NNT}=4.17$ (95\%CI: 2.56, 11.11)). Adverse events were minimal and non-significant.

Conclusions For refractory FD, EA provides significant, clinically relevant symptom relief when added to on-demand gastrocaine. (ChiCTR-IPC-15007109)

\section{IDDF2019-ABS-0119 ENDOSCOPIC ULTRASOUND-GUIDED LEFT LOBE LIVER LESIONS BIOPSY (EUS-LLB) WITH ATYPICAL MALIGNANCIES, AN ALTERNATIVE APPROACH TO RADIOLOGICAL IMAGE-GUIDED BIOPSY}

Adeel Urrehman*, Mohammad Mubarak. Sindh Institute of Urology and Transplantation, Pakistan

\subsection{6/gutjnl-2019-IDDFabstracts.157}

Background Endoscopic ultrasound (EUS) is gaining attraction as an alternative method of biopsy. It offers a more targeted approach for focal lesions in liver especially those areas which are accessible via EUS-guided method.

Methods A total of 7 patients from Feb 2018 till September 2018 underwent EUS-guided Left Lobe lesions biopsies. EUS Guided LLB performed using 22G FNA needle, 2 passes were done with slow pull technique over one minute with 10-15 strokes in each pass to obtained core samples. Duration of procedures ranges from 15-30 minutes.

Results Case 1, 78-Years-old male with a history of gastrectomy $\underline{5}$ years back for biopsy-proven gastric cancer, presented with weight loss and vague epigastric pain, CT scan showed left lobe SOL. Biopsy showed well-differentiated Adenocarcinoma from GI Tract. Case 2, 58-years-old male presented with weight loss and Liver mass on CT Scan. Hepatitis B \& C screen was negative with normal AFP levels. Biopsy revealed Sarcomatoid Carcinoma. Case 3, 53-Years-old male presented with weight loss and abdominal pain. He had multiple lesions identified on CT scan in the liver. Biopsy revealed Neuroendocrine Tumor. Case 4, 35-years-old male presented with abdominal pain. Laboratory tests showed antiHCV reactive with normal AFP levels. CT Scan showed liver lesion suggestive of atypical Hepatocellular carcinoma. Biopsy revealed Smooth Muscle Tumor. As part of workup, he also had gastroscopy and Colonoscopy with no evidence of luminal malignancy. Case 5, 60-years-old female presented with Weight loss, CT scan showed pancreatic malignancy with liver metastasis. Biopsy revealed Metastatic Adenocarcinoma. Case 6, 42-years-old female presented with Obstructive jaundice from Ampullary Carcinoma. She underwent EUS staging which revealed left lobe lesion. Biopsy revealed Metastatic Adenocarcinoma. Case 7, 32-years-old male presented with Weight loss and Obstructive jaundice, CT scan showed left lobe malignancy consistent with Cholangiocarcinoma with normal AFP and CA19-9 levels, his hepatitis B \& C screen negative, the biopsy revealed Lymphoproliferative disease (Lymphoma).

Conclusions EUS-guided LLB is an alternative new technique for biopsy of liver lesions with suspected atypical malignancies. 